\title{
Stochastic differential equations driven by loops
}

\author{
Fabrice Baudoin \\ Department of Mathematics, Purdue University \\ West Lafayette, IN, USA
}

\begin{abstract}
We study stochastic differential equations of the type

$$
X_{t}=x+\sum_{i=1}^{d} \int_{0}^{t} V_{i}\left(X_{s}^{x}\right) \circ d M_{s}^{i}, 0 \leq t \leq T
$$
\end{abstract}

where $\left(M_{s}\right)_{0 \leq s \leq T}$ is a semimartingale generating a loop in the free Carnot group of step $N$ and show how the properties of the random variable $X_{T}^{x}$ are closely related to the Lie subalgebra generated by the commutators of the $V_{i}$ 's with length greater than $N+1$. It is furthermore shown that if $f$ is a smooth function, then

$$
\lim _{T \rightarrow 0} \frac{\mathbb{E}\left(f\left(X_{T}^{x}\right)\right)-f(x)}{T^{N+1}}=\left(\Delta_{N} f\right)(x),
$$

where $\Delta_{N}$ is a second order operator related to the $V_{i}^{\prime} s$.

Keywords: Brownian loops, Carnot groups, Chen development, Holonomy operator, Hörmander's type theorems.

\section{Contents}

1 Introduction

2 Stochastic Differential Equations driven by Brownian Loops and Bridges 5

3 Free Carnot Groups and Hörmander's Type Theorems 10

3.1 Free Carnot groups . . . . . . . . . . . . . . . . . . . 10

3.2 Hörmander's type theorems . . . . . . . . . . . . . . . . 13

4 Stochastic Differential Equations Driven by N-Step Brownian Loops 15

4.1 N-step Brownian Loops . . . . . . . . . . . . . . . . 15

4.2 SDEs Driven by N-Step Brownian Loops . . . . . . . . . . . . . . . 17 


\section{Introduction}

Let us consider a stochastic differential equations on $\mathbb{R}^{n}$ on the type

$$
X_{t}^{x_{0}}=x_{0}+\sum_{i=1}^{d} \int_{0}^{t} V_{i}\left(X_{s}^{x_{0}}\right) \circ d M_{s}^{i}, 0 \leq t \leq T,
$$

where:

1. $x_{0} \in \mathbb{R}^{n}$;

2. $V_{1}, \ldots, V_{d}$ are $C^{\infty}$ bounded vector fields on $\mathbb{R}^{n}$;

3. o denotes Stratonovitch integration;

4. $\left(M_{t}\right)_{0 \leq t \leq T}=\left(M_{t}^{1}, \ldots, M_{t}^{d}\right)_{0 \leq t \leq T}$ is a $d$-dimensional continuous semimartingale.

It is well-known that if $\left(M_{t}\right)_{0 \leq t \leq T}$ is a standard Brownian motion then for any smooth function $f: \mathbb{R}^{n} \rightarrow \mathbb{R}$ we have in $\mathbf{L}^{2}$,

$$
\lim _{t \rightarrow 0} \frac{\mathbf{P}_{t} f-f}{t}=\frac{1}{2}\left(\sum_{i=1}^{d} V_{i}^{2}\right) f
$$

where $\mathbf{P}_{t}$ is the semigroup associated with (1.1) which is defined by

$$
\mathbf{P}_{t} f(x)=\mathbb{E}\left(f\left(X_{t}^{x}\right)\right)
$$

In that case, it is furthermore known from Hörmander's theorem that $\mathbf{P}_{t}$ has a smooth transition kernel with respect to the Lebesgue measure as soon as for all $x_{0} \in \mathbb{R}^{n}$, $\mathfrak{L}\left(x_{0}\right)=\mathbb{R}^{n}$ where $\mathfrak{L}$ is the Lie algebra generated by the vector fields $V_{i}$ 's. So, when $\left(M_{t}\right)_{0 \leq t \leq T}$ is a Brownian motion, the properties of $\left(X_{t}^{x_{0}}\right)_{0 \leq t \leq T}$ are closely related to the diffusion operator $\sum_{i=1}^{d} V_{i}^{2}$ and the Lie algebra $\mathfrak{L}$.

In this paper, we show that there are other choices of the driving semimartingale $\left(M_{t}\right)_{0 \leq t \leq T}$ for which the solution $\left(X_{t}^{x_{0}}\right)_{t \geq 0}$ is naturally associated to other diffusion operators.

Let us roughly describe our approach. The Chen-Strichartz expansion theorem (see [5], [13, [34]) states that, formally, the stochastic flow $\left(\Phi_{t}\right)_{0 \leq t \leq T}$ associated with the stochastic differential equations (1.1) can be written as

$$
\Phi_{t}=\exp \left(\sum_{k \geq 1} \sum_{i_{1}, \ldots, i_{k} \in\{1, \ldots, d\}} F_{i_{1}, \ldots, i_{k}}\left(\int_{0 \leq t_{1} \leq \ldots \leq t_{k} \leq t} \circ d M_{t_{1}}^{i_{1}} \cdots \circ d M_{t_{k}}^{i_{k}}\right)_{\text {anti }}\right), t \leq T
$$


where $F_{i_{1}, \ldots, i_{k}}$ are universal Lie polynomials in $V_{1}, \ldots, V_{d}$, which depend on the choice of a Hall basis in the free Lie algebra with $d$ generators, and $\left(\int_{0 \leq t_{1} \leq \cdots \leq t_{k} \leq t} \circ d M_{t_{1}}^{i_{1}} \cdots \circ d M_{t_{k}}^{i_{k}}\right)_{\text {anti }}$ are universal antisymmetrizations of the iterated integrals of the semimartingale $\left(M_{t}\right)_{t \geq 0}$. Consider now $N \geq 0$, and take for $\left(M_{t}^{1}, \ldots, M_{t}^{d}\right)_{0 \leq t \leq T}$ a $d$-dimensional standard Brownian motion conditioned by

$$
\left(\int_{0 \leq t_{1} \leq \cdots \leq t_{k} \leq T} \circ d M_{t_{1}}^{i_{1}} \cdots \circ d M_{t_{k}}^{i_{k}}\right)_{\text {anti }}=0, i_{1}, \ldots, i_{k} \in\{1, \ldots, d\}, 1 \leq k \leq N .
$$

It is shown that such a process, that we call a $N$-step Brownian loop, is a semimartingale up to time $T$ and can be constructed from a diffusion in the loop space over the free Carnot group of step $N$.

For this choice of $\left(M_{t}\right)_{t \geq 0}$, the Chen development for $\Phi_{T}$ writes

$$
\Phi_{T}=\exp \left(\sum_{k \geq N+1} \sum_{i_{1}, \ldots, i_{k} \in\{1, \ldots, d\}} F_{i_{1}, \ldots, i_{k}}\left(\int_{0 \leq t_{1} \leq \cdots \leq t_{k} \leq t} \circ d M_{t_{1}}^{i_{1}} \cdots \circ d M_{t_{k}}^{i_{k}}\right)_{\text {anti }}\right),
$$

and thus only involves the Lie subalgebra $\mathfrak{L}^{N+1}$, where $\mathfrak{L}$ is the Lie algebra generated by the vector fields $V_{i}$ 's and for $p \geq 2, \mathfrak{L}^{p}$ is inductively defined by

$$
\mathfrak{L}^{p}=\left\{[X, Y], X \in \mathfrak{L}^{p-1}, Y \in \mathfrak{L}\right\}, \mathfrak{L}^{1}=\mathfrak{L} .
$$

Hence, we can expect that the properties of the random variable $X_{T}^{x}$, where $\left(X_{t}^{x}\right)_{0 \leq t \leq T}$ is the solution of (1.1) with initial condition $x$, are closely related to this Lie subalgebra $\mathfrak{L}^{N+1}$.

Precisely, we show that:

- If $f: \mathbb{R}^{n} \rightarrow \mathbb{R}$ is a smooth function which is compactly supported, then in $L^{2}$,

$$
\lim _{T \rightarrow 0} \frac{\mathcal{H}_{T}^{N} f-f}{T^{N+1}}=\Delta_{N} f
$$

where $\Delta_{N}$ is an homogeneous second order differential operator that belongs to the universal enveloping algebra of $\mathfrak{L}^{N+1}$ and $\mathcal{H}_{T}^{N}$ the $N$-step holonomy operator that we define by

$$
\mathcal{H}_{T}^{N+1} f(x)=\mathbb{E}\left(f\left(X_{T}^{x}\right)\right)
$$

- If $\mathfrak{L}^{N+1}=0$, then for all $x \in \mathbb{R}^{n}$, almost surely $X_{T}^{x}=x$; 
- If for all $x \in \mathbb{R}^{n}, \mathfrak{L}^{N+1}(x)=\mathbb{R}^{n}$, then for all $x \in \mathbb{R}^{n}, X_{T}^{x}$ has a smooth density $p_{T}(x)$ with respect to the Lebesgue measure. Moreover, in that case $p_{T}(x) \sim_{T \rightarrow 0}$ $m(x) T^{-\frac{D(x)}{2}}$, where $m$ is a smooth non negative function and $D(x)$ an integer (the graded dimension of $\left.\mathfrak{L}^{N+1}(x)\right)$;

We stress the fact that from a geometrical point of view the family of operators $\left(\Delta_{N}\right)_{N \geq 0}$ is quite interesting and is an important invariant of the intrinsic geometry of the differential system generated by the $V_{i}$ 's. For instance we shall see that, for some positive constant $C$,

$$
\Delta_{1}=C \sum_{i, j=1}^{d}\left[V_{i}, V_{j}\right]^{2}
$$

and, in a way, this operator sharply measures the curvature of the differential system generated by the vector fields $V_{i}$.

Let us mention that, since the seminal work of Rotschild and Stein [33], the study Carnot groups arise naturally in PDE's theory and is now an active research field . Our interest in SDE's driven by loops in Carnot groups originally come from the study of the Brownian holonomy on sub-Riemannian manifolds. The understanding of this holonomy is closely related to the construction of parametrices for hypoelliptic Schrödinger equations (see [?]). The paper is organized as follows. The second section is here for the sake of clarity of the paper since the framework is quite simple but the results already interesting: We study stochastic differential equations driven by Brownian loops. All the results presented in this section will be later generalized. In the third section, we introduce the notion of free Carnot group of step $N$ and define a fundamental diffusion on it. We then study the coupling of this diffusion with the solution of a generic stochastic differential equation driven by Brownian motions. In particular we establish an Hörmander type theorem for the existence of a smooth density for the joint law of this coupling. The fourth section constitutes the heart of this paper and gives the proofs of the results presented above.

Some results of the paper were already announced in the note [3] and the book [5]. 


\section{Stochastic Differential Equations driven by Brownian Loops and Bridges}

We consider first on $\mathbb{R}^{n}$ stochastic differential equations of the type

$$
X_{t}=x_{0}+\sum_{i=1}^{d} \int_{0}^{t} V_{i}\left(X_{s}\right) \circ d P_{s, T}^{i}, t \leq T
$$

where:

1. $x_{0} \in \mathbb{R}^{n}$;

2. $V_{1}, \ldots, V_{d}$ are $C^{\infty}$ bounded vector fields on $\mathbb{R}^{n}$;

3. $\left(P_{t, T}^{1}, \ldots, P_{t, T}^{d}\right)_{0 \leq t \leq T}$ is a given $d$-dimensional Brownian bridge from 0 to 0 with length $T>0$.

Notice that since $\left(P_{t, T}\right)_{0 \leq t \leq T}$ is known to be a semimartingale up to time $T$, the notion of solution for (2.2) is well-defined up to time $T$.

Proposition 2.1 For every $x_{0} \in \mathbb{R}^{n}$, there is a unique solution $\left(X_{t}^{x_{0}}\right)_{0 \leq t \leq T}$ to (2.2). Moreover there exists a stochastic flow $\left(\Phi_{t}, 0 \leq t \leq T\right)$ of smooth diffeomorphisms $\mathbb{R}^{n} \rightarrow$ $\mathbb{R}^{n}$ associated to the equations (2.2).

Proof. We refer to the book of Kunita [24], where the questions of existence and uniqueness of a smooth flow for stochastic differential equations driven by general continuous semimartingales are treated (cf. Theorem 3.4.1. p. 101 and Theorem 4.6.5. p. 173).

The random variable $X_{T}$ where $\left(X_{t}\right)_{0 \leq t \leq T}$ is a solution of (2.2), is of particular interest: It is closely related to the commutations properties of the vector fields $V_{1}, \ldots, V_{d}$. Indeed, let us consider the following family of operators $\left(\mathcal{H}_{T}\right)_{T \geq 0}$ defined on the space of compactly supported smooth functions $f: \mathbb{R}^{n} \rightarrow \mathbb{R}$ by

$$
\left(\mathcal{H}_{T} f\right)(x)=\mathbb{E}\left(f\left(X_{T}^{x}\right)\right), x \in \mathbb{R}^{n} .
$$

Obviously, the family of operators $\left(\mathcal{H}_{T}\right)_{T \geq 0}$ does not satisfy the semigroup property. It is interesting that in some cases, we can explicitly compute $\left(\mathcal{H}_{T}\right)_{T \geq 0}$. 
Theorem 2.2 Assume that the Lie algebra generated by the vector fields $V_{i}$ is two-step nilpotent (that is, any commutator with length greater than 3 is 0 ) then

$$
\mathcal{H}_{T}=\operatorname{det}\left(\frac{T \Omega}{2 \sinh \left(\frac{1}{2} T \Omega\right)}\right)^{\frac{1}{2}}
$$

where $\Omega$ is the $d \times d$ matrix such that $\Omega_{i, j}=\left[V_{i}, V_{j}\right]$.

Before turning to the proof, we mention that the above expression for $\mathcal{H}_{T}$ is understood in the sense of pseudo-differential operators. Namely, the expression

$$
\operatorname{det}\left(\frac{\left(x_{i, j}\right)_{1 \leq i, j \leq d}}{2 \sinh \left(\frac{1}{2}\left(x_{i, j}\right)_{1 \leq i, j \leq d}\right)}\right)^{\frac{1}{2}}
$$

defines an analytic function

$$
\Phi\left(\left(x_{i, j}\right)_{1 \leq i, j \leq d}\right)
$$

and the above theorem says that

$$
\mathcal{H}_{T}=\int_{\mathbb{R}} \frac{d(d-1)}{2} \hat{\Phi}(\xi) e^{i T \sum_{i<j} \xi_{i, j}\left[V_{i}, V_{j}\right]} d \xi
$$

where $\hat{\Phi}$ denotes the Fourier transform of $\Phi$. For further details on pseudo-differential operators we refer to the chapter 7 of [35].

Proof. Itô's formula shows that in that two-nilpotent case,

$$
f\left(X_{T}^{x}\right)=\left(\exp \left(\frac{1}{2} \sum_{1 \leq i<j \leq d}\left[V_{i}, V_{j}\right] \int_{0}^{t} P_{s, T}^{i} d P_{s, T}^{j}-P_{s, T}^{j} d P_{s, T}^{i}\right) f\right)(x) .
$$

But, from Gaveau-Lévy's area formula see [19], if $A$ is a $d \times d$ skew-symmetric matrix valued in a commutative ring, then,

$$
\mathbb{E}\left(e^{i \int_{0}^{T}\left(A P_{s, T}, d P_{s, T}\right)}\right)=\operatorname{det}\left(\frac{t A}{\sin t A}\right)^{\frac{1}{2}} .
$$

This completes the proof.

It seems difficult to find a closed expression for $\mathcal{H}_{T}$ in the general case, we can nevertheless compute a small-time asymptotics: 
Theorem 2.3 Let $f: \mathbb{R}^{n} \rightarrow \mathbb{R}$ be a smooth function which is compactly supported. In $\mathbf{L}^{2}$,

$$
\lim _{T \rightarrow 0} \frac{\mathcal{H}_{T} f-f}{T^{2}}=\frac{1}{24}\left(\sum_{1 \leq i<j \leq d}\left[V_{i}, V_{j}\right]^{2}\right) f .
$$

Proof. We refer to the proof of Theorem 4.8 which is more general. We however show how the constant $\frac{1}{24}$ is obtained. The proof of Theorem 4.8 shows that there is a universal constant $C$ such that

$$
\lim _{T \rightarrow 0} \frac{\mathcal{H}_{T} f-f}{T^{2}}=C\left(\sum_{1 \leq i<j \leq d}\left[V_{i}, V_{j}\right]^{2}\right) .
$$

Since this constant is universal, in order to compute it, it suffices to look at the two-step nilpotent case. In that case, from the previous theorem

$$
\mathcal{H}_{T}=\operatorname{det}\left(\frac{T \Omega}{2 \sinh \left(\frac{1}{2} T \Omega\right)}\right)^{\frac{1}{2}}
$$

Therefore

$$
\mathcal{H}_{T} \sim_{T \rightarrow 0} \operatorname{det}\left(\mathbf{1}-\frac{1}{24} T^{2} \Omega^{2}\right)^{\frac{1}{2}},
$$

and the computation is easily done.

We study now sufficient conditions which ensure that the operator $\mathcal{H}_{T}$ has a smooth kernel (in the two variables) with respect to the Lebesgue measure of $\mathbb{R}^{n}$. To answer this question, it is enough to decide under which conditions the random variable $X_{T}^{x}$ has a smooth density.

On one hand, we have the following result:

Theorem 2.4 Assume that $[\mathfrak{L}, \mathfrak{L}]=0$, then for any solution $\left(X_{t}^{x_{0}}\right)_{0 \leq t \leq T}$ of (2.2) we have almost surely $X_{T}^{x_{0}}=x_{0}$.

Proof. For $i=1, \ldots, d$, let us denote $\left(e^{t V_{i}}\right)_{t \in \mathbb{R}}$ the one-parameter flow associated with the complete vector field $V_{i}$. Since the $V_{i}$ 's are assumed to commute, an iterative application of Itô's formula shows that the process

$$
\left(\left(e^{P_{s, T}^{1} V_{1}} \circ \ldots \circ e^{P_{s, T}^{d} V_{d}}\right)\left(x_{0}\right)\right)_{0 \leq s \leq T}
$$


solves the equation (2.2) with initial condition $x_{0}$. By uniqueness, we have hence

$$
\Phi_{s}\left(x_{0}\right)=\left(e^{P_{s, T}^{1} V_{1}} \circ \ldots \circ e^{P_{s, T}^{d} V_{d}}\right)\left(x_{0}\right), 0 \leq s \leq T .
$$

In particular,

$$
\Phi_{T}\left(x_{0}\right)=x_{0}
$$

which is the expected result.

In general, the weaker condition $[\mathfrak{L}, \mathfrak{L}]\left(x_{0}\right)=0$ is not enough to conclude that for the solution $\left(X_{t}^{x_{0}}\right)_{0 \leq t \leq T}$ of (2.2) we have almost surely $X_{T}^{x_{0}}=x_{0}$. For instance, consider in dimension 2 ,

$$
V_{1}=\left(\begin{array}{l}
1 \\
0
\end{array}\right), \text { and } V_{2}=\left(\begin{array}{l}
0 \\
f(x),
\end{array}\right)
$$

where $f$ is a smooth function whose Taylor development at 0 is 0 (by e.g. $f(x)=$ $\left.e^{-\frac{1}{x^{2}}} \mathbf{1}_{x>0}\right)$. Nevertheless, if the vector fields $V_{i}$ 's are assumed to be analytic on whole $\mathbb{R}^{n},[\mathfrak{L}, \mathfrak{L}]\left(x_{0}\right)=0$ implies that $[\mathfrak{L}, \mathfrak{L}]=0$ and therefore that almost surely $X_{T}^{x_{0}}=x_{0}$.

Theorem 2.5 Assume that $[\mathfrak{L}, \mathfrak{L}]\left(x_{0}\right)=\mathbb{R}^{n}$, then for the solution $\left(X_{t}^{x_{0}}\right)_{0 \leq t \leq T}$ of (2.2) the random variable $X_{T}^{x_{0}}$ has a smooth density with respect to the Lebesgue measure of $\mathbb{R}^{n}$.

Proof. Let us consider the solution $\left(Y_{t}\right)_{t \geq 0}$ of the following stochastic differential equation:

$$
Y_{t}=x_{0}+\sum_{i=1}^{d} \int_{0}^{t} V_{i}\left(Y_{s}\right) \circ d B_{s}^{i}, t \geq 0
$$

where $\left(B_{t}^{1}, \ldots, B_{t}^{d}\right)_{t \geq 0}$ is a $d$-dimensional standard Brownian motion. Since $[\mathfrak{L}, \mathfrak{L}]\left(x_{0}\right)=\mathbb{R}^{n}$, it easily seen that $\left(Y_{t}, B_{t}\right)_{t \geq 0}$ is a diffusion process whose infinitesimal generator satisfies the (strong) Hörmander's condition at $\left(x_{0}, 0\right)$. Therefore, the random variable

$$
\left(Y_{T}, B_{T}\right)
$$

has a smooth density with respect to the Lebesgue measure on $\mathbb{R}^{n} \times \mathbb{R}^{n}$. This implies the existence of a smooth function $p: \mathbb{R}^{n} \rightarrow \mathbb{R}$ such that for all bounded measurable function $f: \mathbb{R}^{n} \rightarrow \mathbb{R}$

$$
\mathbb{E}\left(f\left(Y_{T}\right) \mid B_{T}=0\right)=\int_{\mathbb{R}^{n}} f(y) p(y) d y .
$$

Now, since in law the process $\left(P_{t, T}\right)_{0 \leq t \leq T}$, is identical to the Brownian motion $\left(B_{t}\right)_{0 \leq t \leq T}$ conditioned by $B_{T}=0$, the function $p$ is actually exactly the density of the random variable $X_{T}^{x_{0}}$ where $\left(X_{t}^{x_{0}}\right)_{0 \leq t \leq T}$ is the solution of (2.2) with initial condition $x_{0}$. 
Another proof of this result may be given by using standard Malliavin calculus tools (see chapter 2 of Nualart's book [31, whose notations below are taken).

We work in the $d$-dimensional Wiener space and define the Brownian loop $\left(P_{t, T}^{1}, \ldots, P_{t, T}^{d}\right)_{0 \leq t \leq T}$ as the Wiener integral

$$
P_{t, T}=(T-t) \int_{0}^{t} \frac{d W_{s}}{T-s}, t<T, \text { and } P_{T, T}=0
$$

where $W$ is the $d$-dimensional Wiener process. In this setting, it is not difficult to prove that if $\left(X_{t}\right)_{0 \leq t \leq T}$ is a solution of (2.2), then $X_{T} \in \mathbb{D}^{\infty}$. Moreover, a direct computation shows that for any $0 \leq s \leq T$, the Malliavin derivative is given

$$
\mathbf{D}_{s} X_{T}=\mathbf{J}_{0 \rightarrow T}\left(\mathbf{J}_{0 \rightarrow s}^{-1} \sigma\left(X_{s}\right)-\frac{1}{T-s} \int_{s}^{T} \mathbf{J}_{0 \rightarrow u}^{-1} \sigma\left(X_{u}\right) d u\right),
$$

where $\left(\mathbf{J}_{0 \rightarrow t}\right)_{0 \leq t \leq T}$ is the first variation process defined by

$$
\mathbf{J}_{0 \rightarrow t}=\frac{\partial \Phi_{t}}{\partial x}
$$

and $\sigma$ the $n \times d$ matrix field $\sigma=\left(V_{1}, \ldots, V_{d}\right)$. From this, we can deduce that the Malliavin matrix of $X_{T}$ must be invertible. Indeed, if not, we could find a non zero vector $h \in \mathbb{R}^{d}$ and a finite stopping time $\theta>0$ such that $\mathbf{D}_{s} X_{T} \cdot h=0$ for $0 \leq s \leq \theta$. This would lead to the conclusion that $\left(\mathbf{J}_{0 \rightarrow s}^{-1} \sigma\left(X_{s}\right) \cdot h\right)_{0 \leq s \leq T}$ must be constant.

Observe now that

$$
\mathbf{J}_{0 \rightarrow s}^{-1} V_{i}\left(X_{s}\right)=\Phi_{s}^{*} V_{i}
$$

where $\Phi$ denotes the stochastic flow associated with equation (2.2), and where $\Phi_{s}^{*} V_{i}$ denotes the pull-back action of $\Phi$ on $V_{i}$. Therefore, according to the Itô's formula, we obtain that for $t<\theta$,

$$
\sum_{j=1}^{d} \int_{0}^{t}{ }^{T} h\left(\Phi_{s}^{*}\left[V_{j}, V_{i}\right]\right)\left(x_{0}\right) \circ d P_{s, T}^{j}, \quad i=1, \ldots, d .
$$

is constant. Therefore, for $0 \leq s<\theta$,

$$
{ }^{T} h\left(\Phi_{s}^{*}\left[V_{j}, V_{i}\right]\right)\left(x_{0}\right)=0, \quad i, j=1, \ldots, d .
$$

By applying this at $s=0$, we obtain then

$$
{ }^{T} h\left[V_{j}, V_{i}\right]\left(x_{0}\right)=0, \quad i, j=1, \ldots, d .
$$


New iterations of the Itô's formula show then that, we actually have

$$
{ }^{T} h U\left(x_{0}\right)=0, \quad U \in \mathfrak{L}^{2}\left(x_{0}\right),
$$

so that $h=0$.

We mention that the problem of the existence of densities for stochastic differential equations driven by Brownian bridges was also discussed in [11]. But unlike our case, the existence of the density is discussed for times $t<T$.

\section{Free Carnot Groups and Hörmander's Type Theorems}

In this section we now state some basic facts about Carnot groups.

\subsection{Free Carnot groups}

We introduce the notion of Carnot groups. Such Lie groups appear as tangent spaces to hypoelliptic diffusions (see [4]). For more details on the material presented in this section, we refer to the Chapter 2 of [5]. Let $N \geq 1$. A Carnot group of depth (or step) $N$ is a simply connected Lie group $\mathbb{G}$ whose Lie algebra can be written

$$
\mathcal{V}_{1} \oplus \ldots \oplus \mathcal{V}_{N}
$$

where

$$
\left[\mathcal{V}_{i}, \mathcal{V}_{j}\right]=\mathcal{V}_{i+j}
$$

and

$$
\mathcal{V}_{s}=0, \text { for } s>N
$$

Example 3.1 (Heisenberg Group) The Heisenberg group $\mathbb{H}$ can be represented as the set of $3 \times 3$ matrices:

$$
\left(\begin{array}{lll}
1 & x & z \\
0 & 1 & y \\
0 & 0 & 1
\end{array}\right), \quad x, y, z \in \mathbb{R}
$$

The Lie algebra of $\mathbb{H}$ is spanned by the matrices

$$
D_{1}=\left(\begin{array}{ccc}
0 & 1 & 0 \\
0 & 0 & 0 \\
0 & 0 & 0
\end{array}\right), D_{2}=\left(\begin{array}{ccc}
0 & 0 & 0 \\
0 & 0 & 1 \\
0 & 0 & 0
\end{array}\right) \text { and } D_{3}=\left(\begin{array}{ccc}
0 & 0 & 1 \\
0 & 0 & 0 \\
0 & 0 & 0
\end{array}\right) \text {, }
$$


for which the following equalities hold

$$
\left[D_{1}, D_{2}\right]=D_{3},\left[D_{1}, D_{3}\right]=\left[D_{2}, D_{3}\right]=0
$$

Thus

$$
\mathfrak{h} \sim \mathbb{R} \oplus[\mathbb{R}, \mathbb{R}]
$$

and, therefore, $\mathbb{H}$ is a (free) two-step Carnot group.

Let us now take a basis $U_{1}, \ldots, U_{d}$ of the vector space $\mathcal{V}_{1}$. The vectors $U_{i}$ 's can be seen as left invariant vector fields on $\mathbb{G}$ so that we can consider the following stochastic differential equation on $\mathbb{G}$ :

$$
d \tilde{B}_{t}=\sum_{i=1}^{d} \int_{0}^{t} U_{i}\left(\tilde{B}_{s}\right) \circ d B_{s}^{i}, t \geq 0,
$$

where $\left(B_{t}\right)_{t \geq 0}$ is a standard Brownian motion. This equation is easily seen to have a unique (strong) solution $\left(\tilde{B}_{t}\right)_{t \geq 0}$ associated with the initial condition $\tilde{B}_{0}=0_{\mathbb{G}}$.

Definition 3.2 The process $\left(\tilde{B}_{t}\right)_{t \geq 0}$ is called the lift of the standard Brownian motion $\left(B_{t}\right)_{t \geq 0}$ in the group $\mathbb{G}$ with respect to the basis $\left(U_{1}, \ldots, U_{d}\right)$.

Notice that $\left(\tilde{B}_{t}\right)_{t \geq 0}$ is a Markov process with generator $\frac{1}{2} \sum_{i=1}^{d} U_{i}^{2}$. This second-order differential operator is, by construction, left-invariant and hypoelliptic. For $\left(\tilde{B}_{t}\right)_{t \geq 0}$, we actually have an explicit expression. To give this expression, we first have to introduce some notations. If $I=\left(i_{1}, \ldots, i_{k}\right) \in\{1, \ldots, d\}^{k}$ is a word, we denote $|I|=k$ its length and by $U_{I}$ the commutator defined by

$$
U_{I}=\left[U_{i_{1}},\left[U_{i_{2}}, \ldots,\left[U_{i_{k-1}}, U_{i_{k}}\right] \ldots\right]\right.
$$

The group of permutations of the index set $\{1, \ldots, k\}$ is denoted $\mathfrak{S}_{k}$. If $\sigma \in \mathfrak{S}_{k}$, we denote $e(\sigma)$ the cardinality of the set

$$
\{j \in\{1, \ldots, k-1\}, \sigma(j)>\sigma(j+1)\} .
$$

As a direct consequence of the Chen-Strichartz development theorem (see Proposition 2.3 of [5], or [13], 34]), we have 
Proposition 3.3 We have

$$
\tilde{B}_{t}=\exp \left(\sum_{k=1}^{N} \sum_{I=\left(i_{1}, \ldots, i_{k}\right)} \Lambda_{I}(B)_{t} U_{I}\right), t \geq 0
$$

where:

$$
\Lambda_{I}(B)_{t}=\sum_{\sigma \in \mathfrak{S}_{k}} \frac{(-1)^{e(\sigma)}}{k^{2}\left(\begin{array}{c}
k-1 \\
e(\sigma)
\end{array}\right)} \int_{0 \leq t_{1} \leq \ldots \leq t_{k} \leq t} \circ d B_{t_{1}}^{\sigma^{-1} i_{1}} \circ \ldots \circ d B_{t_{k}}^{\sigma^{-1} i_{k}} .
$$

For instance:

1. The component of the process $\left(\ln \left(\tilde{B}_{t}\right)\right)_{t \geq 0}$ in $\mathcal{V}_{1}$ is simply

$$
\sum_{i=1}^{d} U_{i} B_{t}^{i}, t \geq 0
$$

2. The component in $\mathcal{V}_{2}$ is

$$
\frac{1}{2} \sum_{1 \leq i<j \leq d}\left[U_{i}, U_{j}\right]\left(\int_{0}^{t} B_{s}^{i} d B_{s}^{j}-B_{s}^{j} d B_{s}^{i}\right), t \geq 0 .
$$

The Carnot group $\mathbb{G}$ is said to be free if $\mathfrak{g}$ is isomorphic to the free Lie algebra with $d$ generators with the relations that all brackets of length more than $N$ vanish . In that case, $\operatorname{dim} \mathcal{V}_{j}$ is the number of Hall words of length $j$ in the free algebra with $d$ generators. We thus have, according to Bourbaki [10] (see also Reutenauer [32] pp.96):

$$
\operatorname{dim} \mathcal{V}_{j}=\frac{1}{j} \sum_{i \mid j} \mu(i) d^{\frac{j}{i}}, j \leq N,
$$

where $\mu$ is the Möbius function. We easily deduce from this that when $N \rightarrow+\infty$,

$$
\operatorname{dim} \mathfrak{g} \sim \frac{d^{N}}{N}
$$

An important algebraic point is that, up to an isomorphism there is one and only one free Carnot with a given depth and a given dimension for the basis. Let us denote $m=\operatorname{dim} \mathbb{G}$. Choose now a Hall family and consider the $\mathbb{R}^{m}$-valued process $\left(B_{t}^{*}\right)_{t \geq 0}$ obtained by writing the components of $\left(\ln \left(\tilde{B}_{t}\right)\right)_{t \geq 0}$ in the corresponding Hall basis of $\mathfrak{g}$. It is easily seen that $\left(B_{t}^{*}\right)_{t \geq 0}$ solves a stochastic differential equation that can be written

$$
B_{t}^{*}=\sum_{i=1}^{d} \int_{0}^{t} D_{i}\left(B_{s}^{*}\right) \circ d B_{s}^{i}
$$


where the $D_{i}$ 's are polynomial vector fields on $\mathbb{R}^{m}$ (for an explicit form of the $D_{i}$ 's, which depend of the choice of the Hall basis, we refer to Vershik-Gershkovich [20] pp.27) . With these notations, we have the following proposition (see also [20]).

Proposition 3.4 On $\mathbb{R}^{m}$, there exists a unique group law $\star$ which makes the vector fields $D_{1}, \ldots, D_{d}$ left invariant. This group law is polynomial of degree $N$ and we have moreover

$$
\left(\mathbb{R}^{m}, \star\right) \sim \mathbb{G}
$$

The group $\left(\mathbb{R}^{m}, \star\right)$ is called the free Carnot group of step $N$ over $\mathbb{R}^{d}$. It shall be denoted $\mathbb{G}_{N}\left(\mathbb{R}^{d}\right)$. The process $B^{*}$ shall be called the lift of $B$ in $\mathbb{G}_{N}\left(\mathbb{R}^{d}\right)$.

Remark 3.5 Notice that $\mathbb{G}_{N}\left(\mathbb{R}^{d}\right)$ is, by construction, endowed with the basis of vector fields $\left(D_{1}, \ldots, D_{d}\right)$. These vector fields agree at the origin with $\left(\frac{\partial}{\partial x_{1}}, \cdots, \frac{\partial}{\partial x_{d}}\right)$.

\subsection{Hörmander's type theorems}

Consider now on $\mathbb{R}^{n}$ stochastic differential equations of the type

$$
X_{t}=x_{0}+\sum_{i=1}^{d} \int_{0}^{t} V_{i}\left(X_{s}\right) \circ d B_{s}^{i}, t \geq 0
$$

where:

1. $x_{0} \in \mathbb{R}^{n}$;

2. $V_{1}, \ldots, V_{d}$ are $C^{\infty}$ bounded vector fields on $\mathbb{R}^{n}$;

3. o denotes Stratonovitch integration;

4. $\left(B_{t}^{1}, \ldots, B_{t}^{d}\right)_{t \geq 0}$ is a $d$-dimensional standard Brownian motion.

It is well-known that for every $x_{0} \in \mathbb{R}^{n}$, there is a unique solution $\left(X_{t}^{x_{0}}\right)_{t \geq 0}$ to (3.4) and moreover that there exists a stochastic flow $\left(\Phi_{t}, t \geq 0\right)$ of smooth diffeomorphisms $\mathbb{R}^{n} \rightarrow \mathbb{R}^{n}$ associated to the equations (3.4). Let us denote by $\mathfrak{L}$ the Lie algebra generated by the vector fields $V_{i}$ and for $p \geq 2$, by $\mathfrak{L}^{p}$ the Lie subalgebra defined by

$$
\mathfrak{L}^{p}=\left\{[X, Y], X \in \mathfrak{L}^{p-1}, Y \in \mathfrak{L}\right\}
$$


Moreover if $\mathfrak{a}$ is a subset of $\mathfrak{L}$, we denote

$$
\mathfrak{a}(x)=\{V(x), V \in \mathfrak{a}\}, x \in \mathbb{R}^{n} .
$$

In this framework, we have the following:

Theorem 3.6 Let $x_{0} \in \mathbb{R}^{n}$. If $\mathfrak{L}^{N+1}\left(x_{0}\right)=\mathbb{R}^{n}$, then for any $t>0$, the random variable

$$
\left(X_{t}^{x_{0}}, B_{t}^{*}\right)
$$

has a smooth density with respect to any Lebesgue measure on $\mathbb{R}^{n} \times \mathbb{G}_{N}\left(\mathbb{R}^{d}\right)$, where $\left(X_{t}^{x_{0}}\right)_{t \geq 0}$ is the solution of (3.4) with initial condition $x_{0}$ and $\left(B_{t}^{*}\right)_{t \geq 0}$ the lift of $\left(B_{t}\right)_{t \geq 0}$ in $\mathbb{G}_{N}\left(\mathbb{R}^{d}\right)$.

Proof. With a slight abuse of notation, we still denote $V_{i}$ (resp. $D_{i}$ ) the extension of $V_{i}$ (resp. $\left.D_{i}\right)$ to the space $\mathbb{R}^{n} \times \mathbb{G}_{N}\left(\mathbb{R}^{d}\right)$. The process $\left(X_{t}^{x_{0}}, B_{t}^{*}\right)_{t \geq 0}$ is easily seen to be a diffusion process in $\mathbb{R}^{n} \times \mathbb{G}_{N}\left(\mathbb{R}^{d}\right)$ with infinitesimal generator

$$
\frac{1}{2} \sum_{i=1}^{d}\left(V_{i}+D_{i}\right)^{2} .
$$

Thus, to prove the theorem, it is enough to check the Hörmander's condition for this operator at the point $\left(x_{0}, 0\right)$. Now, notice that $\left[\mathfrak{L}, \mathfrak{g}_{N}\left(\mathbb{R}^{d}\right)\right]=0$, so that

$$
\operatorname{Lie}\left(V_{1}+D_{1}, \ldots, V_{n}+D_{n}\right)\left(x_{0}, 0\right) \simeq \mathfrak{L}^{N+1}\left(x_{0}\right) \oplus \mathfrak{g}_{N}\left(\mathbb{R}^{d}\right)
$$

because $\mathfrak{g}_{N}\left(\mathbb{R}^{d}\right)$ is step $N$ nilpotent. We denoted $\mathbf{L i e}\left(V_{1}+D_{1}, \ldots, V_{n}+D_{n}\right)$ the Lie algebra generated by $\left(V_{1}+D_{1}, \ldots, V_{n}+D_{n}\right)$. The conclusion follows readily.

Example 3.7 For $N=0$, we have $\mathbb{G}_{0}\left(\mathbb{R}^{d}\right)=\{0\}$ and Theorem 3.6 is the classical Hörmander's theorem.

Example 3.8 For $N=1$, we have $\mathbb{G}_{1}\left(\mathbb{R}^{d}\right) \simeq \mathbb{R}^{d}$ and Theorem [3.6 gives a sufficent condition for the existence of a smooth density for the variable

$$
\left(X_{t}^{x_{0}}, B_{t}\right)
$$


Example 3.9 For $N=2$, we have $\mathbb{G}_{2}\left(\mathbb{R}^{d}\right) \simeq \mathbb{R}^{d} \times \mathbb{R}^{\frac{d(d-1)}{2}}$ and Theorem 3.6 gives a sufficient condition for the existence of a smooth density for the variable

$$
\left(X_{t}^{x_{0}}, B_{t}, \wedge B_{t}\right)
$$

where

$$
\wedge B_{t}=\left(\frac{1}{2} \int_{0}^{t} B_{s}^{i} d B_{s}^{j}-B_{s}^{j} d B_{s}^{i}\right)_{1 \leq i<j \leq d}
$$

\section{Stochastic Differential Equations Driven by N-Step Brow- nian Loops}

In this section, we now enter into the heart of our study.

\subsection{N-step Brownian Loops}

On the free Carnot group $\mathbb{G}_{N}\left(\mathbb{R}^{d}\right)$, consider the fundamental process $\left(B_{t}^{*}\right)_{t \geq 0}$ defined as the solution of the stochastic differential equation

$$
B_{t}^{*}=\sum_{i=1}^{d} \int_{0}^{t} D_{i}\left(B_{s}^{*}\right) \circ d B_{s}^{i}, t \geq 0
$$

As a consequence of Hörmander's theorem, the diffusion with generator

$$
\frac{1}{2} \sum_{i=1}^{d} D_{i}^{2}
$$

has a smooth transition kernel $p_{t}(x, y), t>0$ with respect to the Lebesgue measure.

Proposition 4.1 Let $T>0$. There exists a unique $\mathbb{R}^{d}$-valued continuous process $\left(P_{t, T}^{N}\right)_{0 \leq t \leq T}$ such that

$$
P_{t, T}^{N, i}=B_{t}^{i}+\int_{0}^{t} D_{i} \ln p_{T-s}\left(P_{s, T}^{N, *}, 0_{\mathbb{G}_{N}\left(\mathbb{R}^{d}\right)}\right) d s, \quad t<T, \quad i=1, \ldots, d,
$$

where $\left(P_{t, T}^{N, *}\right)_{0 \leq t \leq T}$ denotes the lift of $\left(P_{t, T}^{N}\right)_{0 \leq t \leq T}$ in $\mathbb{G}_{N}\left(\mathbb{R}^{d}\right)$. It enjoys the following properties:

1. $P_{T, T}^{N, *}=0_{\mathbb{G}_{N}\left(\mathbb{R}^{d}\right)}$, almost surely; 
2. for any predictable and bounded functional $F$,

$$
\mathbb{E}\left(F\left(\left(B_{t}\right)_{0 \leq t \leq T}\right) \mid B_{T}^{*}=0_{\mathbb{G}_{N}\left(\mathbb{R}^{d}\right)}\right)=\mathbb{E}\left(F\left(\left(P_{t, T}^{N}\right)_{0 \leq t \leq T}\right)\right)
$$

3. $\left(P_{t, T}^{N}\right)_{0 \leq t \leq T}$ is a semimartingale up to time $T$.

Proof. The construction of the bridge over a given diffusion is very classical and very general (see for example [2], [8], [17] and [22] p.142), so that we do not present the details. The only delicate point in the previous statement is the semimartingale property up to time $T$. In the elliptic case Bismut [8] deals with the end point singularity by proving an estimate of the logarithmic derivatives of the heat kernel. For the heat kernel on Carnot groups, such an estimate can directly be obtained from [23] and 9]. Namely, for $g \in \mathbb{G}_{N}\left(\mathbb{R}^{d}\right), t>0$,

$$
\left|D_{i} \ln p_{t}(g, 0)\right| \leq \frac{C}{\sqrt{t}}
$$

where $C>0$. Now, to prove that $\left(P_{t, T}^{N, *}\right)_{0 \leq t \leq T}$ is a semimartingale up to time $T$, we need to show that for any $1 \leq i \leq d$,

$$
\int_{0}^{T}\left|D_{i} \ln p_{T-s}\left(P_{s, T}^{N, *}, 0\right)\right| d s<+\infty
$$

with probability 1 , which follows therefore from the above estimate.

The semimartingale $\left(P_{t, T}^{N}\right)_{0 \leq t \leq T}$ shall be called a Brownian loop of step $N$.

Example 4.2 The process $\left(P_{t, T}^{1}\right)_{0 \leq t \leq T}$ is simply the d-dimensional Brownian bridge from 0 to 0 with length $T$.

Example 4.3 The process $\left(P_{t, T}^{2}\right)_{0 \leq t \leq T}$ is the d-dimensional standard Brownian motion $\left(B_{t}\right)_{0 \leq t \leq T}$ conditioned by $\left(B_{T}, \wedge B_{T}\right)=0$.

Remark 4.4 Notice that in law,

$$
\left(P_{t, T}^{N}\right)_{0 \leq t \leq T}=\left(\sqrt{T} P_{\frac{t}{T}, 1}^{N}\right)_{0 \leq t \leq T}
$$




\subsection{SDEs Driven by N-Step Brownian Loops}

Consider now on $\mathbb{R}^{n}$ stochastic differential equations of the type

$$
X_{t}=x_{0}+\sum_{i=1}^{d} \int_{0}^{t} V_{i}\left(X_{s}\right) \circ d P_{s, T}^{i, N}, t \leq T
$$

where:

1. $x_{0} \in \mathbb{R}^{n}$;

2. $V_{1}, \ldots, V_{d}$ are $C^{\infty}$ bounded vector fields on $\mathbb{R}^{n}$;

3. $\left(P_{t, T}^{1, N}, \ldots, P_{t, T}^{d, N}\right)_{0 \leq t \leq T}$ is a $d$-dimensional $N$-step Brownian loop from 0 to 0 with length $T>0$.

Proposition 4.5 For every $x_{0} \in \mathbb{R}^{n}$, there is a unique solution $\left(X_{t}^{x_{0}}\right)_{0 \leq t \leq T}$ to 4.8). Moreover there exists a stochastic flow $\left(\Phi_{t}, 0 \leq t \leq T\right)$ of smooth diffeomorphisms $\mathbb{R}^{n} \rightarrow$ $\mathbb{R}^{n}$ associated to the equations [4.8].

We consider now the following family of operators $\left(\mathcal{H}_{T}^{N}\right)_{T \geq 0}$ defined on the space of compactly supported smooth functions $f: \mathbb{R}^{n} \rightarrow \mathbb{R}$ by

$$
\left(\mathcal{H}_{T}^{N} f\right)(x)=\mathbb{E}\left(f\left(X_{T}^{x}\right)\right), x \in \mathbb{R}^{n} .
$$

The operator $\mathcal{H}_{T}^{N}$ shall be called the depth $N$ holonomy operator.

Remark 4.6 Of course, as for $N=1$ which is the case treated in section 2, the operator $\mathcal{H}_{T}^{N}$ does not satisfy a semi-group property.

A relevant intrinsic property of $\mathcal{H}_{T}^{N}$ is the following:

Proposition 4.7 $\mathcal{H}_{T}^{N}$ does not depend on the particular free Carnot group $\mathbb{G}_{N}\left(\mathbb{R}^{d}\right)$.

Proof. Consider the stochastic differential equation

$$
X_{t}=x_{0}+\sum_{i=1}^{d} \int_{0}^{t} V_{i}\left(X_{s}\right) \circ d \tilde{P}_{s, T}^{i, N}, t \leq T
$$

where $\left(\tilde{P}_{t, T}^{1, N}, \ldots, \tilde{P}_{t, T}^{d, N}\right)_{0 \leq t \leq T}$ generates a loop in a free Carnot group $\mathbb{G}$ of step N. Let $\left(B_{t}\right)_{t \geq 0}$ denote a $d$-dimensional standard Brownian motion, and let $\left(B_{t}^{*}\right)_{t \geq 0}\left(\operatorname{resp} .\left(\tilde{B}_{t}\right)_{t \geq 0}\right)$ 
denote a lift in $\mathbb{G}_{N}\left(\mathbb{R}^{d}\right)$ (resp. $\mathbb{G}$ ). Thanks to the proposition 2.10 of [5], there exists a Carnot group isomorphism

$$
\phi: \mathbb{G}_{N}\left(\mathbb{R}^{d}\right) \rightarrow \mathbb{G}
$$

such that $\tilde{B}=\phi\left(B^{*}\right)$. Therefore almost surely, $\tilde{B}_{T}=0$ is equivalent to $B_{T}^{*}=0$ and thus

$$
\left(\tilde{P}_{t, T}^{1, N}, \ldots, \tilde{P}_{t, T}^{d, N}\right)_{0 \leq t \leq T}={ }^{l a w}\left(P_{t, T}^{1, N}, \ldots, P_{t, T}^{d, N}\right)_{0 \leq t \leq T}
$$

Theorem 4.8 Let $f: \mathbb{R}^{n} \rightarrow \mathbb{R}$ be a smooth, compactly supported function. In $\mathbf{L}^{2}$,

$$
\lim _{T \rightarrow 0} \frac{\mathcal{H}_{T}^{N} f-f}{T^{N+1}}=\Delta_{N} f
$$

where $\Delta_{N}$ is a second order differential operator.

Proof. Before we start the proof, let us precise some notations we already used. If $I=$ $\left(i_{1}, \ldots, i_{k}\right) \in\{1, \ldots, d\}^{k}$ is a word, we denote $|I|=k$ its length and by $V_{I}$ the commutator defined by

$$
U_{I}=\left[U_{i_{1}},\left[U_{i_{2}}, \ldots,\left[U_{i_{k-1}}, U_{i_{k}}\right] \ldots\right] .\right.
$$

The group of permutations of the index set $\{1, \ldots, k\}$ is denoted $\mathfrak{S}_{k}$. If $\sigma \in \mathfrak{S}_{k}$, we denote $e(\sigma)$ the cardinality of the set

$$
\{j \in\{1, \ldots, k-1\}, \sigma(j)>\sigma(j+1)\} .
$$

Finally, we denote

$$
\Lambda_{I}\left(P_{., T}^{N}\right)_{t}=\sum_{\sigma \in \mathfrak{S}_{k}} \frac{(-1)^{e(\sigma)}}{k^{2}\left(\begin{array}{c}
k-1 \\
e(\sigma)
\end{array}\right)} \int_{0 \leq t_{1} \leq \ldots \leq t_{k} \leq t} \circ d P_{t_{1}, T}^{N, \sigma^{-1} i_{1}} \circ \ldots \circ d P_{t_{k}, T}^{N, \sigma^{-1} i_{k}} .
$$

Due to the scaling property

$$
\left(P_{t, T}^{N}\right)_{0 \leq t \leq T}=\left(\sqrt{T} P_{\frac{t}{T}, 1}^{N}\right)_{0 \leq t \leq T}
$$

we can closely follow the article of Strichartz [34] (see also Castell [12]), to obtain the following asymptotic development of $f\left(X_{T}^{x}\right)$ :

$$
f\left(X_{T}^{x}\right)=\left(\exp \left(\sum_{k=1}^{2 N+2} \sum_{I=\left(i_{1}, \ldots, i_{k}\right)} \Lambda_{I}\left(P_{., T}^{N}\right)_{T} V_{I}\right) f\right)(x)+T^{\frac{2 N+3}{2}} \mathbf{R}_{2 N+3}(T, f, x),
$$


where the remainder term satisfies when $T \rightarrow 0$,

$$
\sup _{x \in \mathbb{R}^{n}} \sqrt{\mathbb{E}\left(\mathbf{R}_{2 N+3}(T, f, x)^{2}\right)} \leq C
$$

for some non negative constant $C$. By definition of $\left(P_{t, T}^{N}\right)_{0 \leq t \leq T}$, we actually have

$$
\sum_{k=1}^{2 N+2} \sum_{I=\left(i_{1}, \ldots, i_{k}\right)} \Lambda_{I}\left(P_{., T}^{N}\right)_{T} V_{I}=\sum_{k=N+1}^{2 N+2} \sum_{I=\left(i_{1}, \ldots, i_{k}\right)} \Lambda_{I}\left(P_{., T}^{N}\right)_{T} V_{I},
$$

so that

$$
f\left(X_{T}^{x}\right)=\left(\exp \left(\sum_{k=N+1}^{2 N+2} \sum_{I=\left(i_{1}, \ldots, i_{k}\right)} \Lambda_{I}\left(P_{., T}^{N}\right)_{T} V_{I}\right) f\right)(x)+T^{\frac{2 N+3}{2}} \mathbf{R}_{2 N+3}(T, f, x) .
$$

Therefore, since $f$ is assumed to be compactly supported

$$
\mathcal{H}_{T}^{N} f(x)=\mathbb{E}\left(\left(\exp \left(\sum_{k=N+1}^{2 N+2} \sum_{I=\left(i_{1}, \ldots, i_{k}\right)} \Lambda_{I}\left(P_{., T}^{N}\right)_{T} V_{I}\right) f\right)(x)\right)+T^{\frac{2 N+3}{2}} \tilde{\mathbf{R}}_{2 N+3}(T, f, x),
$$

where

$$
\tilde{\mathbf{R}}_{2 N+1}(T, f, x)=\mathbb{E}\left(\mathbf{R}_{2 N+1}(T, f, x)\right) .
$$

Since, by symmetry, we always have

$$
\mathbb{E}\left(\Lambda_{I}\left(P_{., T}^{N}\right)_{T}\right)=0
$$

we have to go at the order 2 in the asymptotic development of the exponential when $T \rightarrow 0$. By neglecting the terms which have order more than $T^{\frac{2 N+3}{2}}$, we obtain

$$
\begin{gathered}
\mathcal{H}_{T}^{N} f(x)=f(x)+\sum_{I=\left(i_{1}, \ldots, i_{N+1}\right)} \frac{1}{2} \mathbb{E}\left(\Lambda_{I}\left(P_{., T}^{N}\right)_{T} \Lambda_{J}\left(P_{., T}^{N}\right)_{T}\right)\left(V_{I} V_{J} f\right)(x)+T^{\frac{2 N+3}{2}} \mathbf{R}_{2 N+3}^{*}(T, f, x), \\
J=\left(j_{1}, \ldots, j_{N+1}\right)
\end{gathered}
$$

where the remainder term $\mathbf{R}_{2 N+3}^{*}(T, f, x)$ is bounded in $\mathbf{L}^{2}$ when $T \rightarrow 0$. This leads to the expected result.

Example 4.9 We have

$$
\Delta_{0}=\frac{1}{2} \sum_{i=1}^{d} V_{i}^{2}
$$

and, as already seen in section 2,

$$
\Delta_{1}=\frac{1}{24} \sum_{1 \leq i<j \leq d}\left[V_{i}, V_{j}\right]^{2}
$$


We now have the following generalization of Theorem 2.4 and Theorem 2.5,

Theorem 4.10 Assume that $\mathfrak{L}^{N+1}=0$, then for any solution $\left(X_{t}^{x_{0}}\right)_{0 \leq t \leq T}$ of (4.8) we have almost surely $X_{T}^{x_{0}}=x_{0}$. On the other hand, assume that $\mathfrak{L}^{N+1}\left(x_{0}\right)=\mathbb{R}^{n}$, then for the solution $\left(X_{t}^{x_{0}}\right)_{0 \leq t \leq T}$ of (4.8) the random variable $X_{T}^{x_{0}}$ has a smooth density with respect to the Lebesgue measure of $\mathbb{R}^{n}$.

Proof. Assume that $\mathfrak{L}^{N+1}=0$, then there exists a smooth map

$$
F: \mathbb{R}^{n} \times \mathbb{G}_{N}\left(\mathbb{R}^{d}\right) \rightarrow \mathbb{R}^{n}
$$

such that, for $x_{0} \in \mathbb{R}^{n}$, the solution $\left(X_{t}^{x_{0}}\right)_{0 \leq t \leq T}$ of the SDE (4.8) can be written

$$
X_{t}^{x_{0}}=F\left(x_{0}, Q_{t, T}^{N}\right)
$$

which implies immediately the expected result.

Assume now that $\mathfrak{L}^{N+1}\left(x_{0}\right)=\mathbb{R}^{n}$. Let us consider the solution $\left(Z_{t}\right)_{t \geq 0}$ of the following stochastic differential equation:

$$
Z_{t}=x_{0}+\sum_{i=1}^{d} \int_{0}^{t} V_{i}\left(Z_{s}\right) \circ d B_{s}^{i}, t \geq 0,
$$

where $\left(B_{t}^{1}, \ldots, B_{t}^{d}\right)_{t \geq 0}$ is a $d$-dimensional standard Brownian motion. From Theorem 3.6, the random variable

$$
\left(Z_{T}, B_{T}^{*}\right)
$$

has a smooth density with respect to any Lebesgue measure on $\mathbb{R}^{n} \times \mathbb{G}_{d, N}$. It implies in the same way as in the proof of Theorem 2.5 that $X_{T}^{x_{0}}$ has a density with respect to the Lebesgue measure because the density of $B_{T}^{*}$ does not vanish at 0 (see [6]).

In the case of the existence of a density for $X_{T}^{x_{0}}$, we can moreover give an equivalent of this density when the length of the loop tends to 0 . To this end, let us precise some notations. We set for $x \in \mathbb{R}^{n}$ and $k \geq N$,

$$
\mathcal{U}_{k}(x)=\operatorname{span}\left\{V_{I}, N \leq|I| \leq k\right\}
$$

In the case where $\mathfrak{L}^{N+1}(x)=\mathbb{R}^{n}$, if $k$ is big enough then $\mathcal{U}_{k}(x)=\mathbb{R}^{n}$. We denote $d(x)$ the smallest integer $k \geq N+1$ for which this equality holds and define the graded dimension

$$
\operatorname{dim}_{\mathcal{H}} \mathfrak{L}^{N+1}(x):=\sum_{k=N+1}^{d(x)} k\left(\operatorname{dim} \mathcal{U}_{k}(x)-\operatorname{dim} \mathcal{U}_{k-1}(x)\right) .
$$


Theorem 4.11 Assume that for any $x \in \mathbb{R}^{n}, \mathfrak{L}^{N+1}(x)=\mathbb{R}^{n}$. Let us denote $p_{T}(x)$ the density of $X_{T}^{x}$ with respect to the Lebesgue measure. We have

$$
p_{T}(x) \sim_{T \rightarrow 0} \frac{m(x)}{T^{\frac{\operatorname{dim}_{\mathcal{H}} \mathfrak{L}^{N+1}(x)}{2}}},
$$

where $m$ is a smooth non negative function.

Proof. Let us, once time again, consider the solution $\left(Z_{t}^{x}\right)_{t \geq 0}$ of the following stochastic differential equation:

$$
Z_{t}^{x}=x+\sum_{i=1}^{d} \int_{0}^{t} V_{i}\left(Z_{s}^{x}\right) \circ d B_{s}^{i}, t \geq 0,
$$

where $\left(B_{t}^{1}, \ldots, B_{t}^{d}\right)_{t \geq 0}$ is a $d$-dimensional standard Brownian motion. From [6] (see also [26]), the density at $(x, 0)$ of the random variable $\left(Z_{T}^{x}, B_{T}^{*}\right)$ behaves when $T$ goes to zero like

$$
\frac{\tilde{m}(x)}{T^{\frac{\operatorname{dim}_{\mathcal{H}} \mathbb{G}_{N}\left(\mathbb{R}^{d}\right)+\operatorname{dim}_{\mathcal{H}} \mathfrak{L}^{N+1}(x)}{2}}},
$$

where $\operatorname{dim}_{\mathcal{H}} \mathbb{G}_{N}\left(\mathbb{R}^{d}\right)=\sum_{j=1}^{N} j \operatorname{dim} \mathcal{V}_{j}$ is the graded dimension of $\mathbb{G}_{N}\left(\mathbb{R}^{d}\right)$, and $\tilde{m}$ a smooth non negative function. Always from [6], the density of the random variable $Y_{T}$ behaves when $T$ goes to zero like

$$
\frac{C}{T^{\frac{\operatorname{dim}_{\mathcal{H}} \mathbb{G}_{N}\left(\mathbb{R}^{d}\right)}{2}}},
$$

where $C$ is a non negative constant. The conclusion follows readily.

\section{References}

[1] G. K. Alexopoulos, N. Lohoué: On the large time behaviour of heat kernels on Lie groups, Duke Math. J. 120 (2003), no. 2, 311-351.

[2] F. Baudoin: Conditioned stochastic differential equations: Theory, Examples and Applications to finance. Stochastic Processes and their Applications, Vol. 100, pp. 109-145, (2002).

[3] F. Baudoin, Equations diffrentielles stochastiques conduites par des lacets dans les groupes de Carnot. (French. English, French summary) [Stochastic differential equations driven by loops in Carnot groups] C. R. Math. Acad. Sci. Paris 338 (2004), no. $9,719-722$. 
[4] F. Baudoin: The tangent space to a hypoelliptic diffusion and applications, Séminaire de probabilités XXXVIII, Springer, (2005).

[5] F. Baudoin: An Introduction to the geometry of stochastic flows, Imperial College Press, (2005).

[6] G. Ben Arous: Développement asymptotique du noyau de la chaleur hypoelliptique sur la diagonale, Annales de l'institut Fourier, tome 39, n¹, p. 73-99, (1989).

[7] G. Ben Arous: Flots et séries de Taylor stochastiques, Journal of Probability Theory and Related Fields, 81, 29-77, (1989).

[8] J.M. Bismut: Large Deviations and the Malliavin calculus, Birkhauser, (1984).

[9] A. Bonfiglioli, E. Lanconelli and F. Uguzzoni: Uniform Gaussian estimates of the fundamental solutions for heat operators on Carnot groups, Adv. Differential Equations 7 (2002), 1153-1192.

[10] N. Bourbaki: Groupes et Algèbres de Lie, Chap. 1-3, Hermann, (1972).

[11] T. Cass, P. Friz, Densities for rough differential equations under Hörrmander's condition. Ann. of Math. (2) 171 (2010), no. 3, 21152141.

[12] F. Castell: Asymptotic expansion of stochastic flows, Prob. Rel. Fields, 96, 225-239, (1993).

[13] K.T. Chen: Integration of paths, Geometric invariants and a Generalized BakerHausdorff formula, Annals of Mathematics, 65, $\mathrm{n}^{\circ} 1,(1957)$.

[14] W.L. Chow: Uber system von lineare partiellen differentialgleichungen erster ordnung, Math. Ann., 117, (1939).

[15] B. Driver, A. Thalmaier: Heat Equation Derivative Formulas for Vector Bundles, J. Func. Anal., 1, 42-108, (2001).

[16] K. D. Elworthy, X. M. Li: Formulae for the derivatives of heat semigroups, J. Func. Anal. 125, 252-286, (1994).

[17] P. Fitzsimmons, J.W. Pitman, M. Yor : Markov bridges, Construction, Palm interpretation and splicing, Seminar on Stochastic Processes, Birkhauser, 101-134, (1993). 
[18] G. B. Folland, E. M. Stein: Hardy spaces on homogeneous groups. Princeton University Press, (1982).

[19] B. Gaveau: Principe de moindre action, propagation de la chaleur et estimées souselliptiques sur certains groupes nilpotents. Acta Math. 139 (1-2), 95-153, (1977).

[20] V. Ya. Gershkovich, A. M. Vershik: Nonholonomic Dynamical Systems, Geometry of Distributions and Variational Problems, In Dynamical Systems VII, Encyclopaedia of Mathematical Sciences, Vol. 16, Eds. V.I. Arnold, S.P. Novikov, (1994).

[21] M. Gordina, Quasi-invariance for the pinned Brownian motion on a Lie group, Stochastic Process. Appl. 104, no. 2, 243-257, (2003).

[22] E.P. Hsu: Stochastic Analysis on manifolds, AMS, Graduate Texts in Mathematics, Volume 38, (2002).

[23] : D.S. Jerison, A. Sanchez-Calle: Estimates for the Heat kernel for a Sum of Squares of Vector Fields, Ind. Math. Journal, Vol. 35, 4, (1986).

[24] H. Kunita: Stochastic Flows and Stochastic Differential Equations, Cambridge studies in advanced mathematics, 24, (1990).

[25] S. Kusuoka: Approximation of Expectation of Diffusion Process and Mathematical Finance, Advanced Studies in Pure Mathematics, Taniguchi Conference on Mathematics Nara, 147-165, (2001).

[26] R. Léandre: Développement asymptotique de la densité d'une diffusion dégénérée, Forum Math. 4, 1, 45-75.

[27] T. Lyons: Differential Equations Driven by Rough Signals.Revista Mathemática Iberio Americana, Vol 14, No 2, 215 - 310, (1998).

[28] T. Lyons, N. Victoir: Cubature on Wiener space, preprint, (2002).

[29] P. Malliavin: Stochastic calculus of variations and hypoelliptic operators. In: Proc. Inter, Symp. on Stoch. Diff. Equations, Kyoto 1976. Wiley, 195-263, (1978).

[30] P. Malliavin: Stochastic Analysis, Grundlehren der mathematischen Wissenschaften, Vol. 313, Springer (1997). 
[31] D. Nualart: The Malliavin calculus and related topics, Springer, Berlin Heidelberg New-York (1995).

[32] C. Reutenauer: Free Lie algebras, London Mathematical Society Monographs, New series 7, (1993).

[33] L.P. Rotschild, E.M. Stein: Hypoelliptic differential operators and Nilpotent Groups, Acta Mathematica, 137, 247-320, (1976).

[34] R.S. Strichartz: The Campbell-Baker-Hausdorff-Dynkin formula and solutions of differential equations, Jour. Func. Anal., 72, 320-345, (1987).

[35] M.E. Taylor: Partial Differential Equations, Qualitative Studies of Linear Equations, Springer, Applied Mathematical Sciences 116,(1996). 